gr-qc/9503003

McGill/95-06

\title{
Black hole entropy without brick walls
}

\author{
Jean-Guy Demers', René Lafrancę and Robert C. Myers'? \\ Department of Physics, McGill University \\ Montréal, Québec, Canada H3A 2T8
}

\begin{abstract}
We present evidence which confirms a suggestion by Susskind and Uglum regarding black hole entropy. Using a Pauli-Villars regulator, we find that 't Hooft's approach to evaluating black hole entropy through a statistical-mechanical counting of states for a scalar field propagating outside the event horizon yields precisely the one-loop renormalization of the standard Bekenstein-Hawking formula, $S=\mathcal{A} /(4 G)$. Our calculation also yields a constant contribution to the black hole entropy, a contribution associated with the one-loop renormalization of higher curvature terms in the gravitational action.
\end{abstract}

\footnotetext{
1jgdemers@hep.physics.mcgill.ca

${ }^{2}$ lafrance@hep.physics.mcgill.ca

${ }^{3}$ rcm@hep.physics.mcgill.ca
} 


\section{Introduction}

It is now over twenty years since Bekenstein introduced the idea that black holes carry an intrinsic entropy proportional to the surface area of the event horizon measured in Planck units, i.e., $\mathcal{A} / \ell_{p}{ }^{2}[1]$. Hawking's discovery[2] that, in quantum field theory, black holes actually generate thermal radiation allowed the determination of a precise formula for this entropy': $S=\mathcal{A} /(4 G)=\mathcal{A} /\left(4 \ell_{p}{ }^{2}\right)$. This Bekenstein-Hawking formula is applicable for any black hole solution of Einstein's equations. Recently, it was shown[4-8] that when gravity is described by a higher-curvature effective action, the Bekenstein-Hawking result is only the leading contribution in an integral of an entropy density over a cross-section of the horizon, i.e., $S=\oint_{\mathcal{H}} d^{2} x \sqrt{h} \rho_{s}$. The contribution of the Einstein-Hilbert action to the entropy density is simply the constant $1 /(4 G)$, which then yields the expected result $\mathcal{A} /(4 G)$. Any higher curvature interactions make additional higher curvature contributions to $\rho_{s}$.

Our understanding of black hole entropy, though, is only within a thermodynamic framework, and despite a great deal of effort, a microphysical understanding of this entropy is still lacking. Many attempts have been made to provide a definition of black hole entropy using statistical mechanics. York [9] suggested that the entropy be considered as the logarithm of the number of ways that the "quantum ergosphere" can be excited during the evaporation of a Schwarzschild black hole into a surrounding thermal bath. This model has the unsatisfying feature of being nonlocal in time since this entropy includes contributions from the entire future evolution of the black hole. Within the membrane paradigm 10, the entropy is associated with the thermal bath of quantum fields perceived by stationary observers under the stretched horizon. In a related approach introduced by 't Hooft 11, the entropy arises from a thermal bath of fields propagating just outside the horizon (see also Ref. [12]). Recently, there has been a great deal of interest in an interpretation of black hole entropy as entanglement entropy. One defines a density matrix $\rho$ by starting with the vacuum state of some quantum field, and tracing over the field degrees of freedom inside the horizon 13. The entropy is then given by the standard formula $S=-\operatorname{Tr}(\rho \log \rho)$. Alternatively, Frolov and Novikov suggest that one should trace out the degrees of freedom external to the horizon 14. Both of the latter approaches should yield the same result as long as the initial global state of the quantum field is a pure state [15]. Further, Kabat and Strassler argued that the density operator constructed with such a trace has a thermal character independent of the details of the quantum field theory [16]. The latter result draws a connection between the entanglement entropy analysis and the two previous approaches.

Another feature common to all four of these calculations is that they yield a black hole entropy proportional to the surface area, but with a divergent coefficient. Thus one must introduce a cut-off to regulate any of these results. For example, 't Hooft introduces a "brick wall", a fixed boundary near the horizon within which the quantum field does not propagate. Susskind and Uglum suggested that these divergences have the correct

\footnotetext{
${ }^{4}$ We adopt the standard conventions of setting $\hbar=c=k_{B}=1$, but we will explicitly retain Newton's constant, $G$, in our analysis. Also, we will employ the metric and curvature conventions of Ref. [3].
} 
form to be absorbed in the Bekenstein-Hawking formula as a renormalization of Newton's constant [17]. Thus these calculations should be regarded as yielding the one-loop correction of quantum field theory to the black hole entropy [18, 19]. Further these authors suggested that the "bare entropy" may have a sensible statistical mechanical interpretation in the context of string theory 17]. The latter conjecture has generated a great deal of interest in understanding black hole entropy in the context of string theory[20].

The purpose of this paper is to investigate the first conjecture of Susskind and Uglum connecting entropy divergences with the renormalization of the coupling constants in the theory. In particular, we test this conjecture by examining a scalar field propagating in a four-dimensional, non-extremal Reissner-Nordstrom black hole background. We begin in section 2 by considering the renormalization of the coupling constants in the gravitational action by a quantum scalar field theory. We regulate the scalar field loops using a PauliVillars scheme, and determine the precise renormalization of Newton's constant. In section 3, we present 't Hooft's approach to calculating the black hole entropy. The advantage of the Pauli-Villars regulator is evident at this stage since it can also be used to implement a cut-off for the entropy calculation. Thus we can remove 't Hooft's brick wall (i.e., the explicit length cut-off in Ref. [11]) and we can compare the results with those found for the effective action. The regulated entropy takes the form: $S=B^{\prime} \mathcal{A} / 4+A^{\prime}$, where $A^{\prime}$ and $B^{\prime}$ are constants which have quadratic and logarithmic dependences on the PauliVillars mass respectively. In the final section, we compare the results of the two previous calculations. We find that $B^{\prime}$ is precisely the same constant found in the renormalization of Newton's constant, while $A^{\prime}$ is related to the renormalization of certain higher-curvature interactions. We conclude with a discussion of these results, including a comment on the extremal Reissner-Nordstrom black hole.

\section{Renormalization of the gravitational action}

In the study of the one-loop effective action 21], one may start with the gravitational action

$$
I_{g}=\int d^{4} x \sqrt{-g}\left[-\frac{\Lambda_{B}}{8 \pi G_{B}}+\frac{R}{16 \pi G_{B}}+\frac{\alpha_{B}}{4 \pi} R^{2}+\frac{\beta_{B}}{4 \pi} R_{a b} R^{a b}+\frac{\gamma_{B}}{4 \pi} R_{a b c d} R^{a b c d}+\ldots\right]
$$

where $\Lambda_{B}$ is the cosmological constant, $G_{B}$ is Newton's constant, while $\alpha_{B}, \beta_{B}$ and $\gamma_{B}$ are dimensionless coupling constants for the interactions which are quadratic in the curvature. The subscript $B$ indicates that all of these constants are the bare coupling constants. The ellipsis indicates that the action may also include other covariant higher derivative interactions, but only those terms explicitly shown will be of interest in the present analysis. We also include the action for a minimally coupled neutral scalar field,

$$
I_{m}=-\frac{1}{2} \int d^{4} x \sqrt{-g}\left[g^{a b} \nabla_{a} \phi \nabla_{b} \phi+m^{2} \phi^{2}\right] .
$$

Here we wish to determine the effective action for the metric which results when in the path integral the scalar field is integrated out. In the present case, this integration is simply 
gaussian, yielding the square root of the determinant of the propagator; the contribution to the effective gravitational action, which is essentially the logarithm of this result, is then given by 21]: $W(g)=-\frac{i}{2} \operatorname{Tr} \log \left[-G_{F}\left(g, m^{2}\right)\right]$. Of course, as it stands, this expression is divergent and must be regulated to be properly defined. The divergences of this one-loop effective action, as well as its metric dependence, are easily identified using an adiabatic expansion for the DeWitt-Schwinger proper time representation of the propagator 22]. This leads to a representation of the scalar one-loop action as an asymptotic series 23]:

$$
W(g)=-\frac{1}{32 \pi^{2}} \int d^{4} x \sqrt{-g} \int_{0}^{\infty} \frac{d s}{s^{3}} \sum_{n=0}^{\infty} a_{n}(x)(i s)^{n} e^{-i m^{2} s}
$$

where the adiabatic expansion coefficients $a_{n}(x)$ are functionals of the local geometry at $x$. Thus, they are local expressions constructed in terms of the metric and the curvature tensor. For example,

$$
\begin{aligned}
& a_{0}(x)=1 \\
& a_{1}(x)=\frac{1}{6} R \\
& a_{2}(x)=\frac{1}{180} R^{a b c d} R_{a b c d}-\frac{1}{180} R^{a b} R_{a b}+\frac{1}{30} \square R+\frac{1}{72} R^{2} .
\end{aligned}
$$

In the present case of four dimensions, the ultraviolet divergences arise as $s \rightarrow 0$ in the first three terms of the series (3).

The effective action may be regulated using many different methods 21, but in the present calculation we adopt a Pauli-Villars regularization scheme 24. In general, such a scheme involves the introduction of a number of fictitious fields with very large masses set by some ultraviolet cut-off scale. Some of these regulator fields are also quantized with the "wrong" statistics, so that their contributions in loops tend to cancel those of the remaining fields. The number, statistics and masses of the regulator fields are chosen in order to render all of the ultraviolet divergences finite. In the present four-dimensional scalar field theory, one introduces five regulator fields: $\phi_{1}$ and $\phi_{2}$, which are two anticommuting fields with mass $m_{1,2}=\sqrt{\mu^{2}+m^{2}} ; \phi_{3}$ and $\phi_{4}$, which are two commuting fields with mass $m_{3,4}=\sqrt{3 \mu^{2}+m^{2}}$; and $\phi_{5}$, which is an anticommuting field with mass $m_{5}=\sqrt{4 \mu^{2}+m^{2}}$. The total action for the matter fields then becomes

$$
I_{m}=-\frac{1}{2} \sum_{i=0}^{5} \int d^{4} x \sqrt{-g}\left[g^{a b} \nabla_{a} \phi_{i} \nabla_{b} \phi_{i}+m_{i}^{2} \phi_{i}^{2}\right]
$$

where the original scalar is included as $\phi_{0}=\phi$ with mass $m_{0}=m$. Now, each field makes a contribution to the effective action as discussed above, except that as a result of the anticommuting statistics for $\phi_{2}, \phi_{3}$ and $\phi_{5}$, their contribution to the effective action has the opposite sign, i.e., $W(g) \simeq+\frac{i}{2} \operatorname{Tr} \log \left[-G_{F}\left(g, m_{i}^{2}\right)\right]$. Let us focus on the divergent terms in eq. (3), since these are the ones for which the regulator fields make significant 
contributions, we then obtain

$$
\begin{aligned}
W_{d i v}= & -\frac{1}{32 \pi^{2}} \int d^{4} x \sqrt{-g} \int_{0}^{\infty} \frac{d s}{s^{3}}\left[a_{0}(x)+i s a_{1}(x)+(i s)^{2} a_{2}(x)\right] \\
& \times\left[e^{-i m^{2} s}-2 e^{-i\left(\mu^{2}+m^{2}\right) s}+2 e^{-i\left(3 \mu^{2}+m^{2}\right) s}-e^{-i\left(4 \mu^{2}+m^{2}\right) s}\right] \\
= & \frac{1}{32 \pi^{2}} \int d^{4} x \sqrt{-g}\left[-C a_{0}(x)+B a_{1}(x)+A a_{2}(x)\right] .
\end{aligned}
$$

In this expression, $A, B$ and $C$ are constants which depend on $m$ and $\mu$, and which diverge for $\mu \rightarrow \infty$ :

$$
\begin{aligned}
A= & \ln \frac{4 \mu^{2}+m^{2}}{m^{2}}+2 \ln \frac{\mu^{2}+m^{2}}{3 \mu^{2}+m^{2}} \\
B=\mu^{2} & {\left[2 \ln \frac{3 \mu^{2}+m^{2}}{\mu^{2}+m^{2}}+4 \ln \frac{3 \mu^{2}+m^{2}}{4 \mu^{2}+m^{2}}\right]+m^{2}\left[\ln \frac{m^{2}}{4 \mu^{2}+m^{2}}+2 \ln \frac{3 \mu^{2}+m^{2}}{\mu^{2}+m^{2}}\right] } \\
C=\mu^{4} & {\left[8 \ln \frac{3 \mu^{2}+m^{2}}{4 \mu^{2}+m^{2}}+\ln \frac{3 \mu^{2}+m^{2}}{\mu^{2}+m^{2}}\right]+2 m^{2} \mu^{2}\left[\ln \frac{3 \mu^{2}+m^{2}}{\mu^{2}+m^{2}}+2 \ln \frac{3 \mu^{2}+m^{2}}{4 \mu^{2}+m^{2}}\right] } \\
& +\frac{m^{4}}{2}\left[\ln \frac{m^{2}}{4 \mu^{2}+m^{2}}+2 \ln \frac{3 \mu^{2}+m^{2}}{\mu^{2}+m^{2}}\right] .
\end{aligned}
$$

Combining the scalar one-loop action with the original bare action in eq. (1), we can identify the renormalized coupling constants in the effective gravitational action

$$
\begin{aligned}
I_{e f f}=I_{g}+ & W \\
=\int d^{4} x & \sqrt{-g}\left[-\frac{1}{8 \pi}\left(\frac{\Lambda_{B}}{G_{B}}+\frac{C}{4 \pi}\right)+\frac{R}{16 \pi}\left(\frac{1}{G_{B}}+\frac{B}{12 \pi}\right)+\frac{R^{2}}{4 \pi}\left(\alpha_{B}+\frac{A}{576 \pi}\right)\right. \\
& \left.\quad+\frac{1}{4 \pi} R_{a b} R^{a b}\left(\beta_{B}-\frac{A}{1440 \pi}\right)+\frac{1}{4 \pi} R_{a b c d} R^{a b c d}\left(\gamma_{B}+\frac{A}{1440 \pi}\right)+\ldots\right]
\end{aligned}
$$

where in the action we discard the total derivative term $\square R$ occurring in $a_{2}$. In particular from eq. (9), we obtain the renormalized Newton's constant

$$
\frac{1}{G_{R}}=\frac{1}{G_{B}}+\frac{B}{12 \pi}
$$

In eq. (9), divergent renormalizations also occur for the cosmological constant $\Lambda_{B}$ and the quadratic-curvature coupling constants $\alpha_{B}, \beta_{B}$ and $\gamma_{B}$. For large values of $\mu$, the constants $A, B$ and $C$ grow to leading order as $\ln (\mu / m), \mu^{2}$ and $\mu^{4}$, respectively, but they also contain subleading and finite contributions. The higher order bare coupling constants (beyond those explicitly shown) would receive finite renormalizations from the finite terms in the one-loop action (3), but they will play no role in the present analysis. 
In the following, we will actually consider a Reissner-Nordstrom black hole, because it provides a more sensitive test of our comparison between the above results and those in 't Hooft's calculation of black hole entropy. Thus our background implicitly includes both a metric and a $U(1)$ gauge potential. Therefore the complete action should be supplemented with a Maxwell term and, in general, additional higher derivative interactions with the metric and gauge fields:

$$
I_{U(1)}=\int d^{4} x \sqrt{-g}\left[-\frac{1}{4} F_{a b} F^{a b}+\delta_{B}\left(F_{a b} F^{a b}\right)^{2}+\lambda_{B} R_{a b} F^{a c} F_{c}^{b}+\ldots\right]
$$

Despite introducing a background gauge field, we consider only a neutral scalar field as above, and therefore, in the effective action, the gauge field interactions are completely unaffected by the scalar one-loop contributions. An obvious extension of the present analysis would be to repeat the calculations for a complex scalar field which couples to the gauge potential.

\section{Entropy calculation}

In Ref. [11], 't Hooft attempted to provide a microphysical explanation of black hole entropy by considering the modes for a scalar field in the vicinity of a black hole. In such a calculation, one finds a divergence in the number of modes because of the infinite blue shift at the event horizon. To regulate his calculation, 't Hooft introduced a "brick wall" cut-off, demanding that the scalar field vanish within some fixed distance of the horizon. 't Hooft introduced this "simple-minded" cut-off as an attempt to mimic what he hoped would be a true physical regulator arising from gravitational interactions. In the present calculation, we will find that the Pauli-Villars regulator introduced in the previous section can be used to implement a covariant cut-off in this entropy calculation, and 't Hooft's brick wall may thereby be removed. In this way, it is possible to make an explicit comparison of the divergences appearing in the entropy and in the effective action.

Our calculation follows that of Ref. [11], but we consider the more general case of a Reissner-Nordstrom (RN) black hole, whose metric can be written in the form

$$
d s^{2}=-\left(1-\frac{r_{-}}{r}\right)\left(1-\frac{r_{+}}{r}\right) d t^{2}+\left[\left(1-\frac{r_{-}}{r}\right)\left(1-\frac{r_{+}}{r}\right)\right]^{-1} d r^{2}+r^{2} d \Omega^{2}
$$

where $d \Omega^{2}$ is the angular line element for a unit two-sphere. We assume a non-extremal RN black hole with $r_{+}>r_{-}$, so that $r=r_{+}$and $r_{-}$correspond to the positions of the outer event horizon and the inner Cauchy horizon, respectively. Results for the Schwarzschild black hole are recovered by simply letting $r_{-} \rightarrow 0$. In this RN background, we consider a minimally coupled neutral scalar field as in eq. (2), which satisfies the Klein-Gordon equation

$$
\left(\square-m^{2}\right) \phi=0 .
$$


As described above, 't Hooft's procedure consists of introducing a brick wall cut-off near the event horizon by setting

$$
\phi(x)=0 \quad \text { for } \quad r \leq r_{+}+h
$$

with $h \ll r_{+}$. To eliminate infrared divergences, a second cut-off is introduced at a large radius $L \gg r_{+}: \phi(x)=0$ for $r \geq L$.

Expanding the scalar field in spherical coordinates $\phi=e^{i E t} Y_{\ell m}(\theta, \varphi) f(r)$, we find that the Klein-Gordon equation becomes

$$
\frac{r^{2} E^{2}}{\left(r-r_{+}\right)\left(r-r_{-}\right)} f(r)+\frac{1}{r^{2}} \partial_{r}\left[\left(r-r_{-}\right)\left(r-r_{+}\right) \partial_{r} f(r)\right]-\left(\frac{\ell(\ell+1)}{r^{2}}+m^{2}\right) f(r)=0 .
$$

In the WKB approximation, one writes $f(r)=\rho(r) e^{i S(r)}$, where $\rho(r)$ is a slowly varying amplitude and $S(r)$ is a rapidly varying phase. To leading order, only first derivatives of the phase are important. In particular, eq. (14) yields the radial wave number $k(r, \ell, E) \equiv \partial_{r} S$ :

$$
k^{2}=\left[\left(1-\frac{r_{-}}{r}\right)\left(1-\frac{r_{+}}{r}\right)\right]^{-2}\left[E^{2}-\left(1-\frac{r_{-}}{r}\right)\left(1-\frac{r_{+}}{r}\right)\left(\frac{\ell(\ell+1)}{r^{2}}+m^{2}\right)\right] .
$$

The number of modes with energy not exceeding $E$ is determined by summing over the degeneracy of the angular modes, and finding the radial mode number by counting the number of nodes in the radial wave function:

$$
\begin{aligned}
g(E) \equiv & \int d \ell(2 \ell+1) \int_{r_{+}+h}^{L} d r \frac{1}{\pi} k(r, \ell, E) \\
= & \frac{1}{\pi} \int_{r_{+}+h}^{L} d r\left[\left(1-\frac{r_{-}}{r}\right)\left(1-\frac{r_{+}}{r}\right)\right]^{-1} \times \\
& \int d \ell(2 \ell+1)\left[E^{2}-\left(1-\frac{r_{-}}{r}\right)\left(1-\frac{r_{+}}{r}\right)\left(\frac{\ell(\ell+1)}{r^{2}}+m^{2}\right)\right]^{1 / 2}
\end{aligned}
$$

Above, the sum over the angular quantum number $\ell$ has also been approximated by an integral, and implicitly this integration runs over the values of $\ell$ for which the square root in the integrand is real.

To determine the thermodynamic properties of this system, we consider the free energy of a thermal ensemble of scalar particles with an inverse temperature $\beta$

$$
\beta F=\sum_{N} \ln \left(1-e^{-\beta E_{N}}\right) .
$$

Using eq. (15) to determine the density of states, we obtain

$$
\begin{aligned}
\beta F= & \int_{0}^{\infty} d E \frac{d g}{d E}(E) \ln \left(1-e^{-\beta E}\right) \\
= & -\frac{\beta}{\pi} \int_{0}^{\infty} \frac{d E}{e^{\beta E}-1} \int_{r_{+}+h}^{L} d r\left[\left(1-\frac{r_{-}}{r}\right)\left(1-\frac{r_{+}}{r}\right)\right]^{-1} \times \\
& \int d \ell(2 \ell+1)\left[E^{2}-\left[\left(1-\frac{r_{-}}{r}\right)\left(1-\frac{r_{+}}{r}\right)\right]\left(\frac{\ell(\ell+1)}{r^{2}}+m^{2}\right)\right]^{1 / 2}
\end{aligned}
$$


where an integration by parts has been used to produce the second line. The integral over $\ell$ can be evaluated to yield

$F=-\frac{2}{3 \pi} \int_{0}^{\infty} \frac{d E}{e^{\beta E}-1} \int_{r_{+}+h}^{L} d r\left[\left(1-\frac{r_{-}}{r}\right)\left(1-\frac{r_{+}}{r}\right)\right]^{-2} r^{2}\left[E^{2}-\left(1-\frac{r_{-}}{r}\right)\left(1-\frac{r_{+}}{r}\right) m^{2}\right]^{3 / 2}$

where the remaining integration is still taken for values where the square root is real. The necessity of the brick wall cut-off is clear at this point, since the integrand diverges with a double pole at the event horizon, i.e., as $r \rightarrow r_{+}$. This divergence is more easily examined by introducing a new variable, $s=1-r_{+} / r$. The free energy is then given by

$$
F=-\frac{2 r_{+}{ }^{3}}{3 \pi} \int_{0}^{\infty} \frac{d E}{e^{\beta E}-1} \int_{h^{\prime}}^{L^{\prime}} \frac{d s}{s^{2}(1-s)^{4}(1-u+u s)^{2}}\left[E^{2}-s(1-u+u s) m^{2}\right]^{3 / 2}
$$

where $u=r_{-} / r_{+}, L^{\prime}=1-r_{+} / L$ and $h^{\prime}=h /\left(r_{+}+h\right) \simeq h / r_{+}$. Thus, for small values of $s$, we have $\int_{h^{\prime}} d s / s^{2} \simeq-1 / h^{\prime}$, which diverges as the brick wall is pulled back to the horizon, i.e., as $h^{\prime} \rightarrow 0$.

Now, rather than considering a single scalar field, we repeat 't Hooft's calculation for the Pauli-Villars regulated field theory introduced in eq. (5). Each of the fields makes a contribution to the free energy as in eq. (17), and the total free energy becomes

$$
\bar{F}=-\frac{2 r_{+}{ }^{3}}{3 \pi} \sum_{i=0}^{5} \Delta_{i} \int_{0}^{\infty} \frac{d E}{e^{\beta E}-1} \int_{h^{\prime}}^{L^{\prime}} \frac{d s}{s^{2}(1-s)^{4}(1-u+u s)^{2}}\left[E^{2}-s(1-u+u s) m_{i}^{2}\right]^{3 / 2}
$$

where $\Delta_{0}=\Delta_{3}=\Delta_{4}=+1$ for the commuting fields, and $\Delta_{1}=\Delta_{2}=\Delta_{5}=-1$ for the anticommuting fields. The free energy of the anticommuting regulator fields comes with a minus sign with respect to the commuting fields, as is required since the role of these fields is to cancel contributions of very high energy modes in the regulated theory. Now, if we examine the divergence of the revised free energy in eq. (18), which arises for small $s$, we find $\sum_{i=0}^{5} \Delta_{i} \int_{h^{\prime}} d s / s^{2}=0$; there is a precise cancellation between the original scalar and the regulator fields. Similarly, we find that a sub-leading logarithmic divergence at small $s$ is also cancelled, since $\sum_{i=0}^{5} \Delta_{i} m_{i}^{2}=0$.

Thus, in the Pauli-Villars regulated theory, we are free to remove 't Hooft's brick wall Setting $h^{\prime}=0$, our expression for the free energy becomes

$$
\bar{F}=-\frac{2 r_{+}{ }^{3}}{3 \pi} \int_{0}^{\infty} \frac{d E}{e^{\beta E}-1} \int_{0}^{L^{\prime}} \frac{d s}{s^{2}(1-s)^{4}(1-u+u s)^{2}} \sum_{i=0}^{5} \Delta_{i}\left[E^{2}-s(1-u+u s) m_{i}^{2}\right]^{3 / 2}
$$

\footnotetext{
${ }^{5}$ Note that our total free energy is actually defined through the limit $h^{\prime} \rightarrow 0$, where the brick wall still played a role in defining the density of states for the individual fields. We assume that the results from this limiting procedure coincide with those arising within the canonical quantization of the Pauli-Villars regulated theory.
} 
Now, integrating over $s$ and $E$, we focus only on the divergent contributions at the horizon and find

$$
\bar{F} \simeq-r_{+}{ }^{3}\left[\frac{\pi}{6(1-u) \beta^{2}} B+\frac{4 \pi^{3}(2-3 u)}{45(1-u)^{3} \beta^{4}} A\right]
$$

where $A$ and $B$ are the same constants given in eqs. (7) and (8), respectively. We emphasize that eq. (20) neglects contributions to the integral which do not diverge as $\mu \rightarrow \infty$. The entropy is then given by

$$
S=\beta^{2} \frac{\partial \bar{F}}{\partial \beta}=r_{+}{ }^{3}\left[\frac{\pi}{3(1-u) \beta} B+\frac{16(2-3 u) \pi^{3}}{45(1-u)^{3} \beta^{3}} A\right] .
$$

Choosing the inverse temperature $\beta$ to correspond to the Hawking temperature of a nonextremal RN black hole, we set

$$
\beta=\frac{4 \pi r_{+}}{1-u}
$$

upon which the entropy (21) becomes

$$
S=\frac{\mathcal{A}}{4} \frac{B}{12 \pi}+\frac{(2-3 u) A}{180}
$$

where $\mathcal{A}=4 \pi r_{+}{ }^{2}$ is the surface area of the event horizon. Thus we see that the entropy contains the constants $A$ and $B$, which give precisely the dependence on the regulator mass $\mu$ appearing in the renormalization of Newton's constant and of the quadratic-curvature coupling constants.

\section{Discussion}

\subsection{Renormalization of the Entropy}

The entropy (22) calculated in section 3, and the standard Bekenstein-Hawking entropy, i.e., $S_{B H}=\mathcal{A} /(4 G)$, are related in a simple way. If the latter is written in terms of the bare Newton's constant, then adding these two entropies yields

$$
\begin{aligned}
S_{B H}+S & =\frac{\mathcal{A}}{4}\left(\frac{1}{G_{B}}+\frac{B}{12 \pi}\right)+\frac{(2-3 u) A}{180} \\
& =\frac{\mathcal{A}}{4 G_{R}}+\frac{(2-3 u) A}{180}
\end{aligned}
$$

where we have used eq. (10) for the renormalized Newton's constant. Hence we find that the first contribution proportional to $B$ in the scalar field entropy provides precisely the one-loop renormalization of the Bekenstein-Hawking entropy. Thus these terms combine in precisely the manner suggested by Susskind and Uglum[17.

Note that we must still account for the constant term proportional to $A$ which appears in eq. (22). Following the recent work on black hole entropy for higher curvature effective 
gravitational actions [4-8], we expect that this constant contribution to the entropy should be related to the quadratic-curvature interactions in the action (1). In particular, we must consider

$$
I_{2}=\int d^{4} x \sqrt{-g}\left[\frac{\alpha_{B}}{4 \pi} R^{2}+\frac{\beta_{B}}{4 \pi} R_{a b} R^{a b}+\frac{\gamma_{B}}{4 \pi} R_{a b c d} R^{a b c d}\right] .
$$

These interactions will modify the standard result for black hole entropy, i.e., the BekensteinHawking entropy, by adding an expression of the form

$$
\Delta S=\oint d^{2} x \sqrt{h}\left[2 \alpha_{B} R+\beta_{B} g_{\perp}^{a b} R_{a b}-\gamma_{B} R^{a b c d} \hat{\varepsilon}_{a b} \hat{\varepsilon}_{c d}\right] .
$$

Here, the integral is evaluated over a space-like cross section of the event horizon, $g_{\perp}^{a b}$ is the metric in the normal subspace to this cross section, and $\hat{\varepsilon}_{a b}$ is the binormal to the cross section - for more details see either Ref. [7] or [8]. When eq. (24) is evaluated in the present background, only the last two terms make a nonvanishing contribution (since $R=0$ for a four-dimensional RN black hole): $\Delta S=-8 \pi u \beta_{B}+16 \pi(1-2 u) \gamma_{B}$. Now, including this contribution along with the previous terms in (23), we obtain the total black hole entropy

$$
\begin{aligned}
S_{\text {total }} & =S_{B H}+\Delta S+S \\
& =\frac{\mathcal{A}}{4}\left(\frac{1}{G_{B}}+\frac{B}{12 \pi}\right)-8 \pi u\left(\beta_{B}-\frac{A}{1440 \pi}\right)+16 \pi(1-2 u)\left(\gamma_{B}+\frac{A}{1440 \pi}\right) \\
& =\frac{\mathcal{A}}{4 G_{R}}-8 \pi u \beta_{R}+16 \pi(1-2 u) \gamma_{R}
\end{aligned}
$$

where, as in eq. (9), we have the renormalized coupling constants: $\beta_{R}=\beta_{B}-\frac{A}{1440 \pi}$ and $\gamma_{R}=\gamma_{B}+\frac{A}{1440 \pi}$. Thus both terms in the scalar field entropy (22) account for precisely the scalar one-loop renormalization of the full black hole entropy. Note that for a Schwarzschild black hole (i.e., that which arises with $u=0$ ), the contribution of $\beta_{R}$ in eq. (26) vanishes because the background curvature would satisfy $R_{a b}=0$. Thus, choosing a RN background allows for a more sensitive comparison between the renormalization of the effective action and 't Hooft's entropy calculation. The appearance of subleading terms in the scalar field entropy (22), and their interpretation in terms of higher curvature contributions to the black hole entropy, have also been discussed in an alternate field-theoretic calculation of black hole entropy in Ref. 25].

A priori, one might not have expected the Pauli-Villars scheme to regulate 't Hooft's entropy calculation at all. In fact, though, not only do we find that the Pauli-Villars scheme regulates the latter calculation, our results are in complete agreement with the suggestion of Susskind and Uglum. The divergences appearing in 't Hooft's statistical-mechanical calculation of black hole entropy are precisely the quantum field theory divergences associated with the renormalization of the coupling constants appearing in the expressions of the entropy. This identification includes both the divergent and finite contributions in the renormalization of the couplings, $G_{B}, \beta_{B}$ and $\gamma_{B}$. This precise equality, including the 
finite terms, occurs because the combinations of masses $\sum \Delta_{i} m_{i}^{2} \ln m_{i}^{2}$ and $\sum \Delta_{i} \ln m_{i}^{2}$ arise naturally in both calculations. We have not considered here any of the remaining finite contributions arising in the free energy (19). It should be possible to identify the corresponding contributions to the black hole entropy with finite renormalization of the higher curvature terms arising from finite terms in the one-loop action (3). There is also a class of contributions to the free energy depending on the infrared cut-off. (Of course, these terms cannot be avoided with the Pauli-Villars regulator, which is an ultraviolet regulator.) To leading order, these infrared terms yield the usual (extensive) free energy for a gas of free scalar particles enclosed in a volume $\frac{4}{3} \pi L^{3}$. There are also lower order terms, which arise due to the curved space-time geometry.

\subsection{Extremal Reissner-Nordstrom}

It is not difficult to repeat our calculations for the case of an extremal RN black hole with $r_{+}=r_{-}$. In this case, 't Hooft's brick wall cut-off leads to ill-defined results 26]. The problem is that the coordinate cut-off, $h$, cannot be converted to a proper length cut-off because any point which is a fixed coordinate distance outside of the extremal horizon is in fact an infinite proper distance from the horizon (on a constant time hypersurface). No such problem arises with the covariant Pauli-Villars regulator. However, precisely at the extremal limit $u=1$, the structure of the small $s$ divergences in eq. (19) changes, and hence we must re-evaluate the integral. We find that the divergent part of the free energy is given by

$$
\bar{F}_{\text {ext }} \simeq-r_{+}{ }^{3}\left[\frac{\pi}{3 \beta^{2}} B+\frac{4 \pi^{3}}{9 \beta^{4}} A\right],
$$

and the entropy which follows is

$$
S_{\text {ext }}=r_{+}{ }^{3}\left[\frac{2 \pi}{3 \beta} B+\frac{16 \pi^{3}}{9 \beta^{3}} A\right] .
$$

Here $A$ and $B$ are the same divergent coefficients (7) and (8) that appear in the scalar one-loop action and in the non-extremal entropy. Hence, with a covariant regulator, we find that the extremal entropy has no stronger divergences than appear in the non-extremal case. In fact, the entire result has essentially the same form as the non-extremal entropy in eq. (21).

To proceed further, one must fix the inverse temperature in eq. (27). Using the standard formula 2], $T=\kappa /(2 \pi)$ where $\kappa$ is the surface gravity, one finds that the temperature is zero since the surface gravity vanishes for the extremal RN black hole. Thus, the inverse temperature $\beta$ diverges, and we find that $S_{\text {ext }}$ vanishes ? This result is in accord with the recent discovery 27] that extremal black holes should have vanishing entropy, since one expects then that the renormalization contribution must also vanish; since the value of entropy is independent of the coupling constants, the renormalized value of zero is still

\footnotetext{
${ }^{6}$ The same is true when using the brick wall regulator 26.
} 
zero. Note that the integral for the free energy in eq. (19) is finite for all $u$ in $0 \leq u \leq 1$. Hence, if one could evaluate the full regulated free energy, the resulting entropy would exhibit a smooth transition from the behavior appearing in eq. (22) for $u<1$ to zero at $u=1$. Further the transition should occur for $u-1=O(m / \mu)$. Thus we speculate that the total black hole entropy for which we are calculating the one-loop renormalization should also make a smooth transition to $S=0$ in the extremal limit, but that the precise details of the transition would depend on the ultraviolet characteristics of the underlying theory of quantum gravity.

On the other hand, the recent investigations of extremal black holes [27] also suggest that an extremal black hole can be in equilibrium with a heat bath of an arbitrary temperature. Hence, one might consider leaving the inverse temperature arbitrary in eq. (27). In this case, one has the curious result that $S_{\text {ext }}$ appears to represent the renormalization of some finite entropy expression for an extremal RN black hole. For example, the first term in eq. (27) would represent the renormalization of $S=\frac{\mathcal{A}}{4 G} \frac{8 \pi r_{+}}{\beta}$. Previous calculations have given no indication that such an entropy arises for extremal black holes, and so one may conclude that one must use $\beta \rightarrow \infty$ in this case. Alternatively, it may be that 't Hooft's model does not capture the full physics of extremal black holes, and that the correct result should still be $S_{\text {ext }}=0$ even with a nonvanishing temperature.

\subsection{On-shell/Off-shell}

With few exceptions 25, 28], discussions and derivations of black hole entropy focus on black hole backgrounds which are saddle-points of the gravitational action under investigation, i.e., backgrounds which are solutions of the equations of motion (see, for example, Refs. [4, 5, 6]). We will now discuss this point in the context of the present calculation. Our RN background, which includes both the metric of eq. (12) and, implicitly, a vector potential $A=Q /(4 \pi r) d t$, solves the Einstein-Maxwell equations with $4 \pi r_{+} r_{-}=G Q^{2}$. Demanding that the background be a solution of the equations arising from the total effective action would require that the renormalized cosmological constant vanish, that (most of) the renormalized coupling constants for the higher derivative interactions vanish】, and that the renormalized Newton's constant appear in the above equation with $r_{+}, r_{-}$and $Q$.

For the present calculations, first of all we note that this question of whether or not the background solved any equations of motion is irrelevant for 't Hooft's entropy calculations in section 3. Further, note that if the RN black hole is a solution of the renormalized equations of motion, it cannot at the same time solve the bare equations of motion (which may appear more appropriate for section 3). In our discussion, though, we use the usual

\footnotetext{
${ }^{7}$ Since $R=0$ for the four-dimensional RN background, the metric would still solve the higher derivative equations of motion if $\alpha_{R}$ was nonvanishing. Further, because the combination $R_{a b c d} R^{a b c d}-4 R_{a b} R^{a b}+R^{2}$ is a topological density in four dimensions which does not affect the equations of motion, $\beta_{R}=-4 \gamma_{R}$ would ensure that the RN black hole solves the renormalized equations of motion. Generically, though, one expects for the higher derivative interactions in the effective action (including those not explicitly shown in eqs. (9) and (11) that the corresponding coupling constant must be set to zero to ensure that the $\mathrm{RN}$ background is a solution.
} 
entropy expressions (i.e., the Bekenstein-Hawking formula, and the higher curvature corrections in eq. (24)) to assign our (single) background a black hole entropy within both the renormalized and the bare theories. Thus the present calculations treat these entropy formulas as being valid off-shell, i.e., valid for backgrounds which do not satisfy the equations of motion. This possibility is suggested in Ref. [25], which presented a derivation of black hole entropy which made no explicit use of the equations of motion.

\subsection{Robustness}

One would like to know whether the present results hold for arbitrary field theories coupled to gravity, rather than for just a minimally coupled scalar field. One simple extension of our calculations would be to consider a non-minimally coupled scalar field. The original matter action in eq. (2) is then extended to

$$
I_{m}^{\prime}=-\frac{1}{2} \int d^{4} x \sqrt{-g}\left[g^{a b} \nabla_{a} \phi \nabla_{b} \phi+m^{2} \phi^{2}+\xi R \phi^{2}\right] .
$$

It is well known 21] that the additional coupling of the scalar field to the curvature modifies the adiabatic expansion coefficients in eq. (田), and therefore it affects the renormalizations of the bare coupling constants. For example, eq. (10) for the renormalized Newton's constant is replaced by

$$
\frac{1}{G_{R}}=\frac{1}{G_{B}}+\frac{B}{2 \pi}\left(\frac{1}{6}-\xi\right)
$$

On the other hand, if we repeat the calculation of section 3 for the new scalar field theory, we find that the resulting entropy is completely unchanged. The new coupling constant $\xi$ enters the new equation of motion, $\left(\square-m^{2}-\xi R\right) \phi=0$, which replaces eq. (13). The remainder of the calculation is unmodified, though, because $R=0$ for the background RN metric. Given that Newton's constant is renormalized as in eq. (28), the entropy in eq. (22), which is independent of $\xi$, does not properly account for the renormalization of the Bekenstein-Hawking formula.

We see this failure as a limitation of 't Hooft's model for the calculation of black hole entropy. It is clear that this model does not capture the full physics of the problem. For example, the free energy in eq. (20) does not contain a quartic divergence as would be expected from the renormalization of the cosmological constant in eq. (9). This omission can be traced to the fact that eq. (16) does not include a contribution from the zero point energies. Of course, neglecting this contribution is entirely appropriate for a leading order WKB calculation.

The Euclidean path integral would provide an alternate approach to this calculation ${ }^{\mathbb{8}}$. In fact, this approach provides a more rigorous framework to study black hole entropy. We

\footnotetext{
${ }^{8}$ At this point, we note that in introducing the regulator fields in eq. (18), it may appear that choosing $\Delta_{i}=-1$ and a Bose-Einstein distribution (rather than a Fermi-Dirac distribution) for the anticommuting fields is somewhat arbitrary. In the Euclidean path integral, though, both of these choices are completely fixed.
} 
are presently adapting our analysis to the Euclidean path integral, and expect that it will

yield the correct renormalization of black hole entropy, even for non-minimally coupled scalar fields.

\section{Acknowledgments}

This research was supported by NSERC of Canada and Fonds FCAR du Québec. We would like to thank Cliff Burgess, Keith Dienes, Noureddine Hambli, Ted Jacobson and Alex Marini for useful conversations. RCM would also like to thank the Institute for Advanced Study for its hospitality while a portion of this paper was written.

\section{References}

[1] J.D.Bekenstein, Lett. Nuov. Cim. 4 (1972) 737; Phys. Rev. D7 (1973) 2333; Phys. Rev. D9 (1974) 3292.

[2] S.W. Hawking, Commun. Math. Phys. 43 (1975) 199.

[3] R.M. Wald, General Relativity (University of Chicago Press, Chicago, 1984).

[4] T. Jacobson and R.C. Myers, Phys. Rev. Lett. 70 (1993) 3684, hep-th/9305016.

[5] R.M. Wald, Phys. Rev. D48 (1993) 3427, gr-qc/9307038.

[6] M. Visser, Phys. Rev. D48 (1993), 5697, gr-qc/9307194.

[7] T. Jacobson, G. Kang and R.C. Myers, Phys. Rev. D49 (1994) 6587, gr-qc/9312023.

[8] V. Iyer and R.M. Wald, Phys. Rev. D50 (1994) 846, gr-qc/9403028.

[9] J.W. York, Phys. Rev. D28 (1983) 2929.

[10] W.H. Zurek and K.S. Thorne, Phys. Rev Lett. 54 (1985) 2171; K.S. Thorne, W.H. Zurek and R.H. Price, in Black Holes: The Membrane Paradigm, eds. K.S. Thorne, R.H. Price and D.A. MacDonald (Yale University Press, New Haven, 1986), p. 280.

[11] G. 't Hooft, Nucl. Phys. B256 (1985) 727.

[12] R.B. Mann, L. Tarasov and A. Zelnikov, Class. Quant. Grav. 9 (1992) 1487.

[13] L. Bombelli, R.K. Koul, J. Lee and R.D. Sorkin, Phys. Rev. D34 (1986) 373.

[14] V. Frolov and I. Novikov, Phys. Rev. D48 (1993) 4545, gr-qc/9309001.

[15] M. Srednicki, Phys. Rev. Lett. 71 (1993) 666, hep-th/9303048. 
[16] D. Kabat and M.J. Strassler, Phys. Lett. B329 (1994) 46, hep-th/9401125.

[17] L. Susskind and J. Uglum, Phys. Rev. D50 (1994) 2700, hep-th/9401070.

[18] C. Callan and F. Wilczek, Phys. Lett. B333 (1994) 55, hep-th/9401072.

[19] T. Jacobson, Black Hole Entropy and Induced Gravity, Maryland preprint grqc/9404039; Phys. Rev. D50 (1994) 6031, gr-qc/9407022.

[20] L. Susskind, Some Speculations About Black Hole Entropy in String Theory, Rutgers University preprint RU-93-44, hep-th/9309145; M. McGuigan, Phys. Rev. D50 5225 (1994), hep-th/9406201; J. L. F. Barbón, Phys. Lett. B339 (1994) 41, hepth/9406209; A. Dabholkar, Nucl. Phys. B439 (1995) 650, hep-th/9408098; Phys. Lett. B347 (1995) 222, hep-th/9409158; D. A. Lowe and A. Strominger, Phys. Rev. D51 (1995) 1793, hep-th/9410215.

[21] See, for example, N.D. Birrell and P.C.W. Davies, Quantum Fields in Curved Space, (Cambridge University Press, Cambridge, 1982).

[22] B.S. DeWitt, Phys. Rep. 19C (1975) 297; B.S. DeWitt, 'The dynamical theory of groups and fields' in Relativity, groups and topology, eds. B.S. DeWitt and C. DeWitt (New York: Gordon \& Breach, 1965); J. Schwinger, Phys. Rev. 82 (1951) 664.

[23] P.B. Gilkey, J. Diff. Geom. 10 (1975) 601; S.M. Christensen, Phys. Rev. D14 (1976) 2490; B.S. DeWitt, Phys. Rep. C19 (1975) 296.

[24] C. Bernard and A. Duncan, Ann. Phys. (NY) 107 (1977) 201; T.S. Bunch, S.M. Christensen and S.A. Fulling, Phys. Rev. D18 (1978) 4435.

[25] S.N. Solodukhin, Phys. Rev D51 (1995) 609, hep-th/9407001; Phys. Rev D51 (1995) 618, hep-th/9408068; D.V. Fursaev and S.N. Solodukhin, On One-Loop Renormalization of Black-Hole Entropy, preprint E2-94-462, hep-th/9412020.

[26] A. Ghosh and P. Mitra, Entropy for Extremal Reissner-Nordstrom Black Holes, preprint SISSA-172-94-EP, hep-th/9411128; S.P. de Alwis and N. Ohta, On the Entropy of Quantum Fields in Black Hole Backgrounds, preprint COLO-HEP-347, hepth/9412027.

[27] G.W. Gibbons and R.E. Kallosh, Phys. Rev. D51 (1995) 2839, hep-th/9407118; S.W. Hawking, G.T. Horowitz and S.F. Ross, Phys. Rev. D51 (1995) 4302, gr-qc/9409013; C. Teitelboim, Phys. Rev. D51 (1995) 4315, hep-th/9410103; J. Preskill, P. Schwarz, A. Shapere, S. Trivedi and F. Wilczek, Mod. Phys. Lett. A6 (1991) 2353.

[28] B.F. Whiting and J.W. York, Phys. Rev. Lett. 61 (1988) 1336; J. Louko and B.F. Whiting, Class. Quant. Grav. 9 (1992) 457; S. Carlip and C. Teitelboim, The OffShell Black Hole, preprint IASSNS-HEP-93/84, gr-qc/9312002. 